\title{
TESTING AUTOMATION WITH COMPUTER AIDED TEST CASE GENERATION
}

\author{
Tapio Heikkilä, Petri Tenno and Jarmo Väänänen \\ NetHawk Solutions Oy, Elektroniikkatie 2 A \\ FIN-90570 Oulu, FINLAND \\ tapio.heikkila@nethawk.fi
}

\begin{abstract}
This paper considers techniques to increase the level of automation in testing object oriented software systems. The focus is in utilizing software requirements specifications; for this a computer aided test case generation tool prototype is introduced. The tool uses software requirements specifications in the form of system level message sequences (UML collaboration models) and constraint descriptions of the input and output variables (attribute constraints in UML class models).
\end{abstract}

Key words: software testing, test case generation, testing automation, UML

\section{INTRODUCTION}

Software testing provides a great potential for improving the efficiency of software development. Software testing means a wider set of systematic methods and techniques for organizing the tests, test system, and carrying out the actual tests. While looking for methods of improvement, a process oriented development does not alone lead us to desired results but automation with appropriate strategy and software tools are implied.

Here we consider techniques to increase the level of automation in testing object oriented software systems. In the following chapters software development and testing processes are reviewed and possibilities to automate the testing are discussed. We propose to utilize software requirements specifications for improving the efficiency of testing GUI based software. For this a computer aided test case generation tool prototype is introduced, to be used together with a commercial GUI test driver. The tool has been

The original version of this chapter was revised: The copyright line was incorrect. This has been corrected. The Erratum to this chapter is available at DOI: 10.1007/978-0-387-35497-2_31 
developed initially for improving the efficiency within internal software development of the NetHawk group (NetHawk, 2001). The test case generation tool uses software requirements specifications, given in the form of system level message sequences, expressed as UML collaboration models, and constraint descriptions of the input and output variables, expressed as attribute constraints in UML class models. We consider certain testing techniques, originating in the equivalence partitioning principle (Kit, 1995) as the basis for test case design for system testing. Our target is to provide a software tool to increase the level of automation in the testing, especially by decreasing the level of manual programming of testing scripts. However, we are not targeting to full automation, neither in the sense of having automated all possible test cases nor in the sense of having a tool without human interaction during test planning and design. We assume to be dealing with component based software, e.g., with a user interface composed of GUI components, because the test case execution mechanism is based on a GUI test driver integrating to the software under test through the windows API, typical for GUI components.

\section{SOFTWARE DEVELOPMENT AND TESTING PROCESSES}

A clear and sound software process model is necessary for systematic software development, including testing. The process model can interconnect the oftware development activities into the testing activities by a revised waterfall model, i.e., the V-model (Fewster and Graham, 1999) or even adding the storage \& retrieval of the re-usable components; X-model, (Hodgson, 1990) into the process model. V- and X-models cover the whole software process but are abstract in the sense that they do not provide details about the stages of development, and further on, the entry criteria (or inputs) and exit criteria (or outputs) of the different phases. There are a number models focusing on these details, especially for object oriented development using Unified Modeling Language (UML) as a representation framework, though without the dynamics and synchronization of the development and testing activities. Although using UML as a notational framework, their development phases do have different types of UML models as outcomes. In our case we have followed the Real-Time UML. Real-Time UML is dividing the development into three phases (Douglass, 1998)

1. Requirements Analysis: Context modelling, Use Case modelling, Scenario modelling

2. Analysis: Defining object structure, Defining object behaviour 
3. Design: Architectural design (system and processor wide design), Mechanistic design (inter-object design), Detailed design (intra-object design)

We are considering here the system testing phase, which needs information from requirements analysis and analysis.

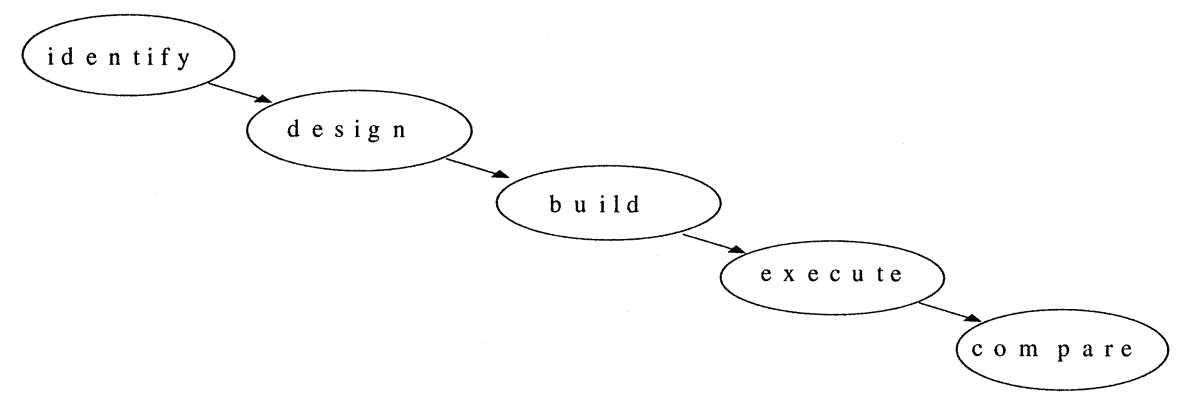

Figure 1. General testing process (adapted from (Fewster and Graham, 1999)).

The testing (also integration and module testing) is composed of different phases (see fig. 1):

identify test conditions (determine, "what" can be tested; which test cases) design test cases, determine, "how" the "what" is to be tested.

build test cases (implement script, data etc.) by preparing program code for tests as test scripts or special test software (in $\mathrm{C} / \mathrm{C}++$, Java, etc) with test inputs and expected outcomes,

execute test cases in the test environment and log the actual outcomes, and compare test case outcomes to expected outcomes.

We are considering here especially the automatic building of test cases for system testing.

\section{SOFTWARE TESTING TECHNIQUES AND TESTING AUTOMATION}

There are many tools for software testing, varying from general test management tools to measuring of the dynamic performance of the executed programs. Different testing tools and their location in the V-model is presented in figure 1.

Test identification and design are at their best based on a well defined design methodology or technique. We are considering the three techniques described in further details below.

Equivalence partitioning is a systematic process that identifies, on the basis of whatever information is available, a set of interesting classes of input conditions to be tested, where a class is representative (or covers) a 
large set of other possible tests. If partitioning is applied to the product under test, the product is going to behave in much the same way for all members of the class (Kit, 1995).

Boundary-value analysis is a variant and refinement of equivalence partitioning with two major differences. First, rather than selecting any element in the equivalence class as being representative, elements are selected such that each edge of the equivalence class is the subject of a test. Second, rather than focusing exclusively on input conditions, output conditions are also explored by defining output equivalence classes (Kit, 1995).

Extended Use Case Test is a test design pattern to develop applicationspecific tests (Binder, 1999). Extended Use Case Test applies when most, if not all of the essential requirements for the SUT can be expressed as use cases. It is possible to develop test cases by imagining specific ideas for a use case and corresponding expected results.

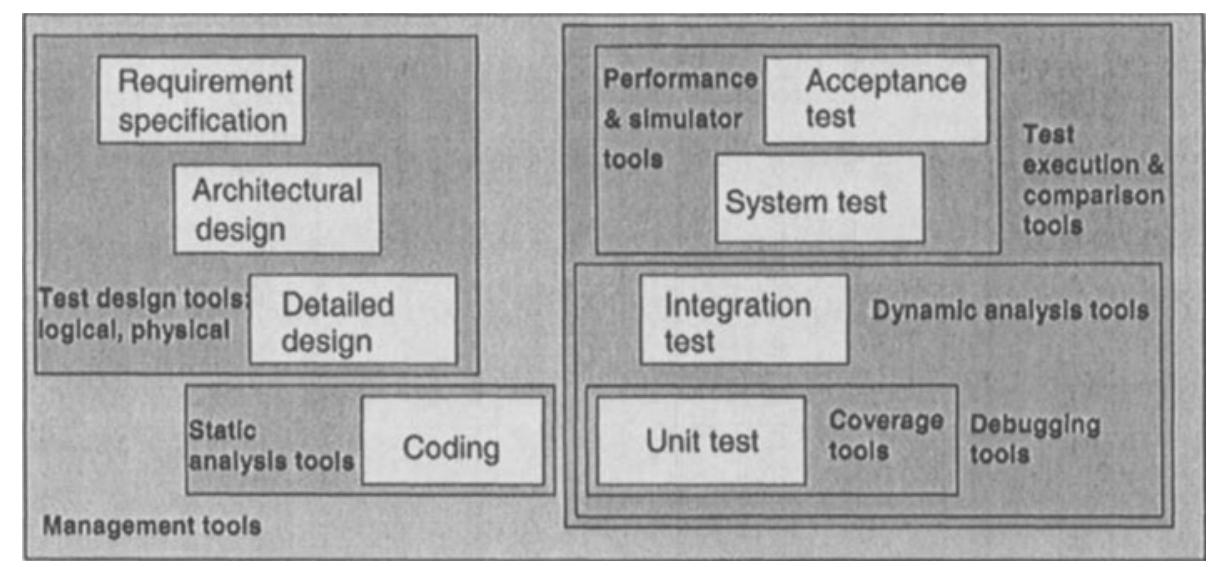

Figure 2. Testing tools vs. software development (revised from Fewster and Graham, 1999).

Component Based Development (CBD) (Carney, 1999) has been leading the way for efficient software development, especially for distributed software systems. Sometimes CBD introduces confusion because of varying interpretations about what is a component and what is not. The definitions given in the following are followed here.

- "A Component is an implementation entity, including source code units, binary code (the output of a translator), and linked, loadable executable files" (Binder, 1999)

- "Components are the packaging construct for the final application. They control, how the application can be delivered and distributed (Kozaczynski and Christerson, 2000)" 
Software components are often more or less present in modern software development i.e., within an intra-application composition (e.g., Java beans, Arnold and Gosling, 1999) or inter-application composition using distribution frameworks like Common Object Request Broker Architecture (CORBA, Mowbray and Ruh, 1997), Enterprise Java Beans (EJB; Shannon et. al., 2000), or Distributed Component Object Model (DCOM; Kirtland, 1999). Component based software development offers an additional dimension considering the possibilities for testing automation. Components, or their containers implement a special, common interface, and at the best the tools implement the required testing points (for control or observation) within these interfaces and in application independent points (parts of the component model).

\section{COMPUTER AIDED TEST GENERATION (CATG)}

Computer Aided Test Generation (CATG) tools are used to automate the "design" and "build" phases of the testing process. CATG tools create abstract or executable test cases based on software requirements or test design. We have developed a test case generator tool prototype, where we create executable test cases based on the requirements and functional specifications of the software under test without needing to explicitly design the test cases. The test case generator takes as inputs a UML Rational Rose ${ }^{\circledR}$ model (Rational, 2001): requirements specifications as system level sequence diagrams, class model of the GUI objects (PCO's) and constraints for the GUI object attributes.

The test case generator parses the UML model and extracts the GUI object data, and creates and shows to the user a table of input attribute values or value constraints acquired from the constraint descriptions in the class model, and a table of output values or value constraints correspondingly. The system can also variate the inputs, i.e., create combinations of input values or constraints for the test cases. The user must create value sets (or rows) of the expected output values by picking combinations of the output value sets. Based on these value sets the system creates final outputs in the form of executable scripts for a GUI test driver environment (WinRunner ${ }^{\circledR}$ TSL scripts, WinRunner, 2001) and also corresponding script and rows in the constraint tables (input values; expected outcomes).

The strategy for identifying and creating test cases lies in the idea of utilizing the requirements specifications of the software. Either in the requirements specification phase or the analysis phase the components in the user interface are modeled as instances of the available GUI API classes. 
Here we consider those GUI components, which have communication with the "operator" actor. In the class model each of the attributes of these objects should get a constraint description, in fact for valid and for invalid values. These constraints are considered here as the sets of equivalence classes for each input and output variable, expressed as algebraic constraints. These can be described e.g., in the Object Constraint Language (Warmer, 1999). During test case generation, an arbitrary value satisfying the constraint (e.g., inside a range) is created and taken into the set of input values of that input variable. Output values are also described as equivalence classes with corresponding constraints. If the output value is a range, then comparison of the actual output value is compared to the range (inside / outside), and if the output value is a single value, then the actual output value is compared to this (equal/unequal). It should be noted, that the constraints could cover both "valid" and "invalid" classes of the input values, or only "valid" ones in which case the "invalid" values could be composed of the complements of the valid value sets. The course of developing the test cases is illustrated within the screenshots in the figure 3 .

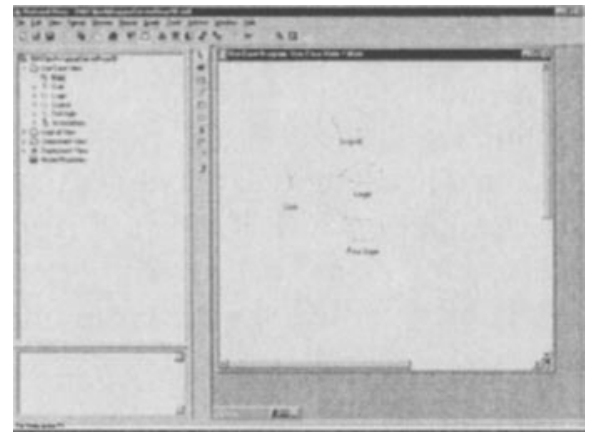

(a) Identify Test Cases:

Req specs in UML (Use Case models)

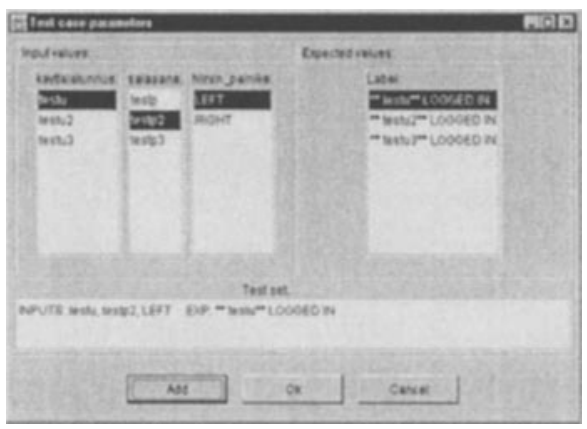

(c) Build Test Cases:

Semiautomatic test case generation

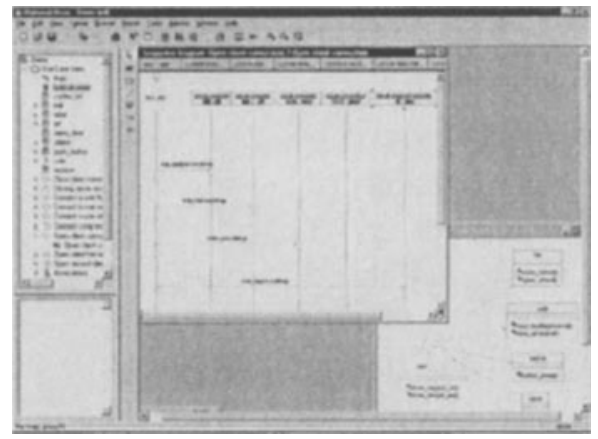

b) Design Test Cases:

Req specs \& analysis in UML (interaction models, class/object models)

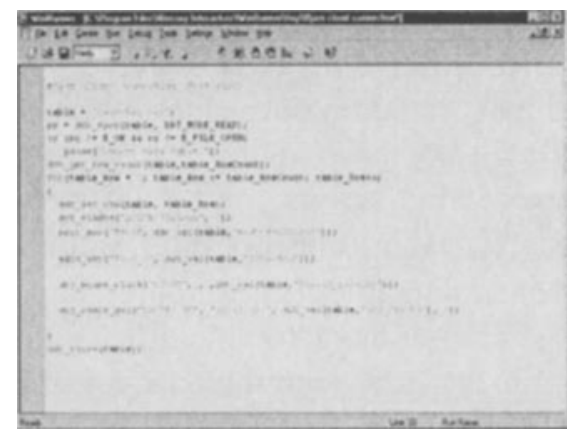

(d) Execute Test Cases:

An executable TSL script 


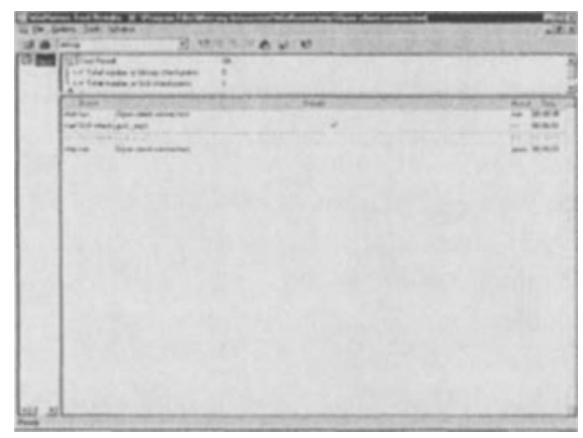

(e) Compare results: WinRunner $®$ report on a test run

Figure 3. CATG based testing: identify (a), design (b), build (c), execute (d), compare (e).

\section{CONCLUSIONS}

In this paper techniques and methods concerning software development and software testing automation were discussed. Computer Aided Test Case Generation was considered in more details and a tool prototype was presented. The tool is utilizing UML specifications of the software, developed in a commercial CASE-tool. The tool supports some most general system testing techniques, originating from the equivalence partitioning method.

\section{REFERENCES}

Arnold K., Gosling J., The Java Programming Language. Addison Wesley, Massachusetts, UA, 1997. 444p.

Binder R. V., Testing Object-Oriented Systems: Models, Patterns, and Tools. Addison Wesley, 1999. 1248 p.

Carney D., COTS Product Evaluation and System Design, COTS Spot - Volume 2, issue 1, March 1999. $6 \mathrm{p}$.

Douglass, B. P., Real-Time UML - Developing Efficient Objects for Embedded Systems. Addison Wesley, Reading, Massachusetts, USA 1998, 400 p.

Dustin E., Rashka J. S., Paul J., Automated Software Testing: Introduction, Management, and Performance. July 1999, Addison-Wesley Pub Co.

Fewster M., Graham D., Software Test Automation - Effective use of test execution tools. Addison Wesley, Oxford, 1999. 574 p.

Grabowski J., Wiles A., Willcock C., Hogrefe D., On the design of the new testing language TTCN-3. 13th IFIP International Workshop on Testing Communicating Systems'

(Testcom 2000), Ottawa, 29.8.2000-1.9.2000, Kluwer Academic Publishers, August 2000.

Hodgson R., The X-Model: A Process Model for Object Oriented Software Development. The $4^{\text {th }}$ International Conference on Software Engineering and Its aApplications. Toulouse, France, December $9-13,1991$. 
Jaaksi A., Aalto J.-M., Aalto A., Vättö K., Tried \& True Object development-Industry-proven Approaches with UML. Cambridge University Press, New York, USA, 1999, 315 p.

Kirtland M., Designing Component Based Applications. Microsoft Press, Redmond, Washingotn, USA, 1999. $427 \mathrm{p}$.

Kit E., Software Testing in the Real World: Improving the Process, ACM Press Books, November 1995, Addison-Wesley, 252 p.

Kozaczynski W., Christerson M., Rose, Rational's Architecture Practice, and Architectureal Reuse. Rose Architect, Winter 2000, pp. 4 - 6.

Kruchten P., The Rational Unified Process, An Introduction, Second edition, Addison Wesley, Cambridge, MA, USA, 2000, 298 p.

McDonnell S., Rapoid Development, Taming Wild Software Schedules. Microsoft Press, Redmond, Washington, 1996. 648 p.

Mowbray T. J., Ruh W. A., Inside CORBA, Distribted Object Standards and Applications. Addison Wesley, Massachusetts, 1997. 376 p.

NetHawk Oy, company info; in "http://www.nethawk.fi"

Newmarch J. D., Testing Java Swing-based Applications. In http://pandonia.canberra.edu.au/java/replayJava/paper.html

Orbiter adds gravity to CORBA. In http://www.nccglobal.com/testing/tmntimes/issue2/tmntimes2_4.htm

Rational Rose, http://www.rational.com

Rosenberg D., Scott K., Use Case Driven Object Modeling with UML - A Practical Approach, Addison Wesley, Reading, Massachusetts, USA, 1999, 172 p.

Schmuller J., Teach Yourself UML in 24 Hours, SAMS Publishing,, Indianapolis, USA, 1999, 422 p.

Segue Launches CORBA products. In http://www.segue.com/html/s_news/press_releases/s_CORBA.htm

Shannon B. et al., Java 2 Platform, Enterprise Edition: Platform and Component Specifications. Addison-Wesley, 2000.

Warmer, The Object Constraint Language . Precise Modeling with UML. Addison Wesley, Reading, Massachisetts, USA, $1999.112 \mathrm{p}$.

WinRunner GUI Testing SW, in http://www.mercuryinteractive.com

\section{Acknowledgements}

This work has been financially supported by the ETX Development Program of the Technology Development Centre of Finland (TEKES), which is greatly acknowledged by the authors. 